\title{
Gain Flattening of L-band EDFA -Raman Hybrid Amplifier
}

\author{
Avneet Kour \\ M.Tech Student \\ Department of Electronics and Communication \\ PEC University of Technology \\ Chandigarh, India
}

\author{
Neena Gupta, PhD \\ Professor \\ Department of Electronics and Communication \\ PEC University of Technology \\ Chandigarh, India
}

\begin{abstract}
A design of L-band EDFA cascaded with distributed Raman amplifier has been optimized so as to improve the gain, noise figure and gain variation. Firstly, the L- band EDFA system has been designed and the effect of change in input power, change in the length of the doped fiber and the use of various pumps on EDFA has been examined. It has been observed that gain obtained is maximum with a low noise figure at $1480 \mathrm{~nm}$ pump with the length of doped fiber of $150 \mathrm{~m}$. Secondly, this system is then cascaded with distributed Raman amplifier (DRA) to form a hybrid optical amplifier (HOA) so as to further improve the gain, noise figure and gain flatness. The HOA system has the best flat gain spectrum of $\pm 0.73 \mathrm{~dB}$ over the bandwidth of $42 \mathrm{~nm}$ with the gain of $29.43 \mathrm{~dB}$ and the noise figure of $4.403 \mathrm{~dB}$.
\end{abstract}

\section{Keywords}

EDFA (Erbium doped fiber amplifier), L-band, Gain, Noise figure (NF), Hybrid optical amplifier (HOA), Distributed Raman amplifier (DRA), Gain flatness.

\section{INTRODUCTION}

Erbium doped fiber amplifier (EDFA) is the most commonly used amplifier in optical communication system. But, with the growth of data traffic and ever increasing demand of internet, there is the need of extending the operating band from C-band $(1525 \mathrm{~nm}-1565 \mathrm{~nm})$ to L-band (1565-1610nm) [1]. The C-band has been of great importance to optical communication because it provides efficient optical amplification with low losses [2] but the L-band is a region of even lower losses [3]. EDFA is less efficient in the L-band so different techniques are used for better amplification. Earlier, the L-band EDFA was used in combination with optical filters [4-5], Raman amplifier, wavelength dependent splitters. But optical filters and wavelength dependent splitters introduce losses and flexibility issues. L-band EDFA with distributed Raman amplifier (Hybrid optical amplifier) [6] can provide wide gain in the required wavelength but till now, gain obtained of hybrid optical amplifier (HOA) is small with low noise figure and more gain variation.

\section{SYSTEM DESIGN}

The HOA system is simulated in VPI PHOTONICS version 9.0. In proposed HOA-WDM system, 100 channels with emission frequency of $184 \mathrm{e} 12 \mathrm{~Hz}$ and channel spacing of 0.8 $\mathrm{nm}$ has been investigated. The HOA-WDM system consists of EDFA section cascaded with distributed Raman amplifier section. The first stage (Erbium doped fiber amplifier section) is forward pumped by $1480 \mathrm{~nm}$ pump with pump power of $100 \mathrm{mw}$ with the length of doped fiber $=150 \mathrm{~m}$. The second stage (distributed Raman amplifier) is pumped by counter pumping scheme. The effect of various pumps on gain, noise figure and gain variation has been analyzed in the counter pumping scheme.

The first stage is excited by WDM combination. The optical coupler combines the input signal and pump signal and is propagated by Erbium doped fiber amplifier. TESTSETAMPLIFIER module is used around the first stage amplifier system. Isolator is used to prevent the back propagation of the signals which causes the reflections resulting in unstability of the whole system. The signal amplified by the first stage is then propagated by the second stage consisting of distributed Raman amplifier which is pumped by counter propagation technique with multiple pumps. TestSetAmplifier modules are used in the second stage amplifier system and the whole amplifier system. In the end, the combined signal is fed into the optical spectrum analyzer to analyze the performance of optical spectrum.

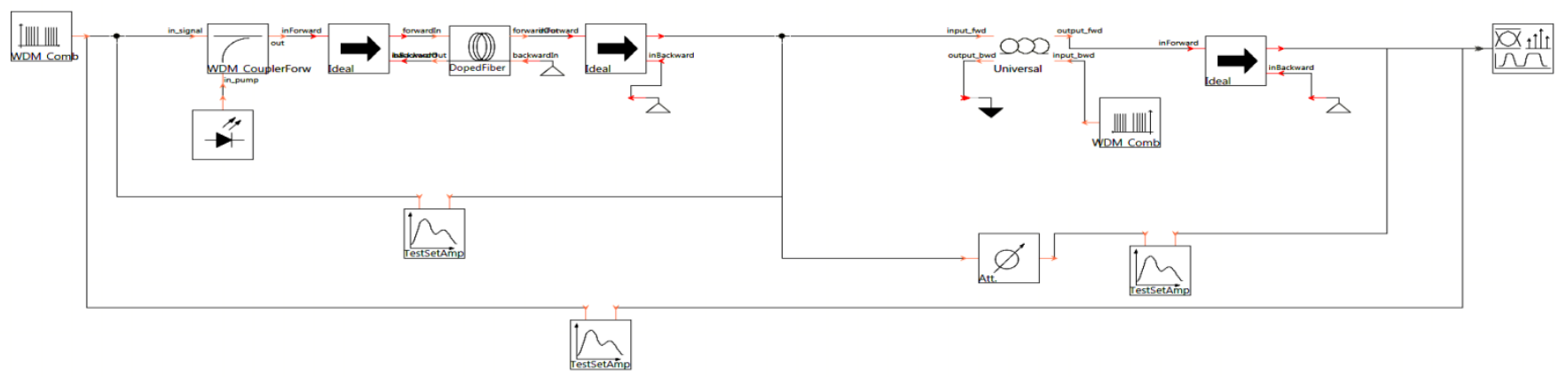

Figure 1: Block diagram of HOA system with multiple pumps in counter pumping technique 


\section{RESULTS}

After simulating setup, the results have been plotted and tabulated with different parameter values of simulation results i.e. gain, noise figure with counter pumping technique. Also, different components use the different operational parameters.

To find out the channel spacing, the linewidth conversion formula [7] is used:

$$
\Delta f=\frac{c}{\lambda^{2}} \Delta \lambda
$$

Where $\mathrm{c}$ is the velocity of light, $\Delta \mathrm{f}$ is the laser linewidth in frequency domain, $\Delta \lambda$ is the laser line width in spectral domain.

Figure 1 shows the schematic of hybrid amplifier. Individual test sets are used to assess the performance of EDFA stage and Raman stage. The stages are:

- A forward pumped EDFA stage

- A multiple backward pumped Raman stage with dedicated fiber

The pumps of Raman amplifier are distributed in wavelength to provide the gain over a wide range.The WDM parameters are shown in table 1 .

\section{Table 1: WDM_COMB Parameters}

\begin{tabular}{|c|c|}
\hline Emission frequency & $184 \mathrm{e} 12 \mathrm{~Hz}$ \\
\hline Channel spacing & $100 \mathrm{e} 9 \mathrm{~Hz}$ \\
\hline Channel power & $1 \mathrm{e}-6 \mathrm{~W}$ \\
\hline No. of channels & 100 \\
\hline
\end{tabular}

In the first stage ( Erbium doped fiber amplifier section), the gain obtained is best at $1480 \mathrm{~nm}$ pump so here $1480 \mathrm{~nm}$ pump is used in spite of $980 \mathrm{~nm}$ pump as it has higher optical conversion efficiency. The length of doped fiber is chosen as $150 \mathrm{~m}$ as the operating band is L- band which requires long length of the doped fiber. The EDFA parameters used in simulation setup is shown in table 2 .

Table 2: EDFA Parameters

\begin{tabular}{|c|c|}
\hline Model Type & Er two level \\
\hline Pump wavelength & $1480 \mathrm{~nm}$ \\
\hline Average pump power & $100 \mathrm{e}-3 \mathrm{~W}$ \\
\hline Length of doped fiber & $150 \mathrm{~m}$ \\
\hline
\end{tabular}

In the second stage (Raman amplifier section), Raman amplifier is backward pumped with dedicated universal fiber. The length of universal fiber is chosen as 45km.TestSetAmplifier is placed across this section which displays On/Off gain and lumped equivalent noise figure simultaneously. An attenuator is placed before the Preinput of TestSetAmplifier and set its attenuation to that of the fiber when the pump is off $(45 \mathrm{~km} * 0.1 \mathrm{e}-03 \mathrm{~dB} / \mathrm{km})$. Thus, the TestSetAmplifier is fooled into thinking that it is measuring a lumped amplifier at the end of an attenuating fiber. The Raman fiber parameters used in simulation setup is shown in table 3 .
Table 3: Raman Fiber Parameter

\begin{tabular}{|c|c|}
\hline Length of Raman fiber & $45 \mathrm{~km}$ \\
\hline Attenuation & $0.1 \mathrm{e}-3 \mathrm{~dB} / \mathrm{km}$ \\
\hline Raman scattering & On \\
\hline Pump power & $100 \mathrm{~mW}$ \\
\hline
\end{tabular}

Table 4: Effect of various pump wavelengths of Raman on gain and noise figure of hybrid amplifier

\begin{tabular}{|c|c|c|c|c|c|c|c|}
\hline $\begin{array}{c}\text { S. } \\
\text { No }\end{array}$ & \multicolumn{4}{|c|}{ Pump wavelengths (nm) } & $\begin{array}{l}\text { Gain } \\
(\mathrm{dB})\end{array}$ & $\begin{array}{l}\text { Noise } \\
\text { figure } \\
(\mathrm{dB})\end{array}$ & $\begin{array}{l}\text { Gain } \\
\text { variati } \\
\text { on } \\
(\mathrm{dB})\end{array}$ \\
\hline 1. & 1448 & 1450 & 1452 & 1454 & 20.63 & 4.4053 & 3.58 \\
\hline 2. & 1462 & 1464 & 1466 & 1468 & 23.73 & 4.4066 & 5.96 \\
\hline 3. & 1470 & 1472 & 1474 & 1476 & 26.25 & 4.4058 & 6.49 \\
\hline 4. & 1482 & 1484 & 1486 & 1488 & 28.84 & 4.4039 & 4.77 \\
\hline 5. & 1492 & 1494 & 1496 & 1498 & 30.18 & 4.4023 & 1.44 \\
\hline 6. & 1502 & 1504 & 1506 & 1508 & 29.43 & 4.4035 & 0.73 \\
\hline 7. & 1512 & 1514 & 1516 & 1518 & 27.53 & 4.4065 & 0.86 \\
\hline 8. & 1522 & 1524 & 1526 & 1528 & 26.24 & 4.4088 & 0.87 \\
\hline
\end{tabular}

As seen from Table 4, when Raman amplifier is backward pumped with $1502,1504,1506,1508 \mathrm{~nm}$, the gain obtained is $29.43 \mathrm{~dB}$ with the noise figure of $4.403 \mathrm{~dB}$. The system has the best flat gain spectrum of $\pm 0.73 \mathrm{~dB}$ over the bandwidth of $42 \mathrm{~nm}$.However, in all the cases, the noise figure approximately remains constant.

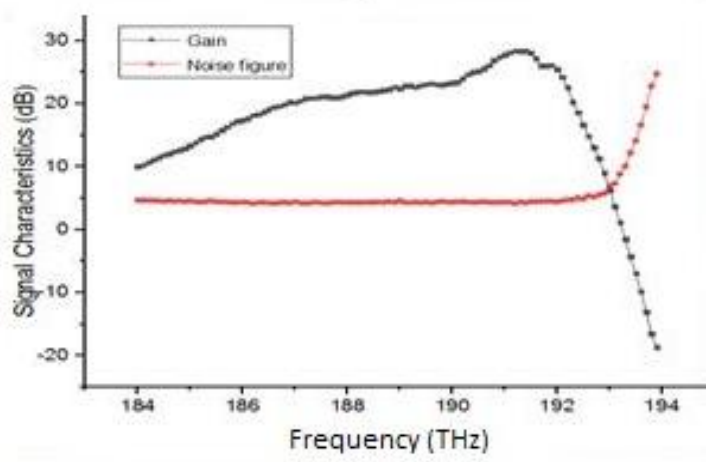

Figure 2: Gain and noise figure analysis of $\mathrm{L}$ - band EDFA

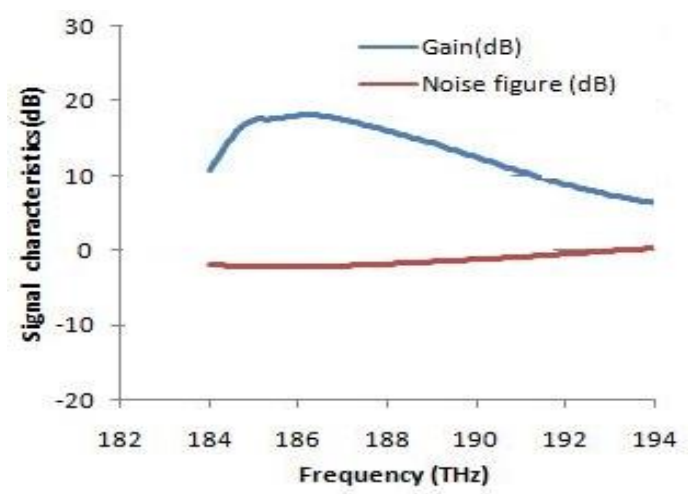

Figure 3: Gain and noise figure of Raman amplifier pumped at 1502, 1504, 1506, 1508nm 
The above figure shows the gain and noise figure of multipump Raman amplifier as measured by TestSetAmplifier. An attenuator with the equivalent attenuation of the unpumped fiber is used to obtain the (pump) on-off gain and equivalent lumped amplifier noise figure.

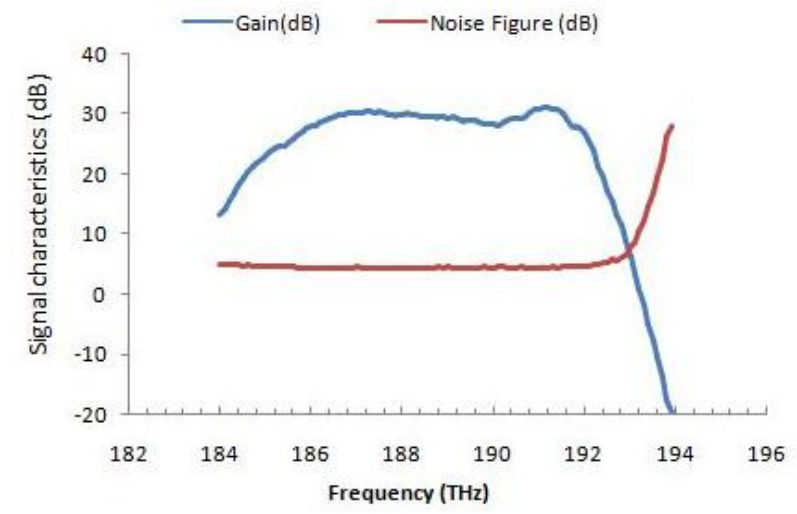

Figure 4: Overall gain and NF of HOA pumped at 1502, 1504, 1506, 1508nm

The above figure shows the overall gain of $29.43 \mathrm{~dB}$ and noise figure of $4.403 \mathrm{~dB}$ with the gain variation of $\pm 0.73 \mathrm{~dB}$.

\section{CONCLUSION}

A Hybrid amplifier has been proposed using EDFA- Raman amplifier. The length of the doped fiber is $150 \mathrm{~m}$ with input channel power of 1e-6W. When backward pumped with 1502 , $1504,1506,1508 \mathrm{~nm}$ in counter pumping scheme, the gain of HOA is $29.43 \mathrm{~dB}$ with the noise figure of $4.403 \mathrm{~dB}$. The system has a flat gain spectrum of $\pm 0.73 \mathrm{~dB}$ over the bandwidth of $42 \mathrm{~nm}$. A low gain variation with better gain and better noise figure is obtained without using any costly gain flattening techniques. This work can be further extended to hybrid optical amplifiers combined with other gain media including combination of Raman amplifiers with fibers doped with different rare earth media (e.g., $\mathrm{Nd}$ and $\mathrm{Yb}$ ).

\section{REFERENCES}

[1] Jiuru Yang, Xiangyu Meng, and Chunyu Liu. "Accurately control and flatten gain spectrum of L-band erbium doped fiber amplifier based on suitable gainclamping." Optics \& Laser Technology 78, pp.74$78,2016$.

[2] Meenakshi Sharma and Vivek Ruder Sharma. "Gain flattening of EDFA in C-band using RFA for WDM application." In Signal Processing and Integrated Networks (SPIN), 2015 2nd International Conference on, pp. 346-351. IEEE, 2015.

[3] B. A. Hamida, S. M. Azooz, A. A. Jasim, T. Eltaif, S. Khan, H. Ahmad, and S. W. Harun. "Flat-gain wide-band erbium doped fiber amplifier with hybrid gain medium." Optik-International Journal for Light and Electron Optics 127, no. 5, pp.2481-2484,2016.

[4] M. Jung, H.Choi, D. Lee, J. Seong, S.Jung, S. Lee. “ Demonstrartion of highly efficient flat gain L-band Erbium doped fiber amplifiers by incorporating a fiber bragg grating.” IEEE Photon, pp. 1258-1260, 2002.

[5] S. W. Harun, S. K. Low, P. Poopalan, and H. Ahmad. "Gain clamping in L-band Erbium-doped fiber amplifier using a fiber Bragg grating." IEEE photonics technology letters 14, no. 3,pp. 293-295,2003.

[6] Simranjit Singh and R. S. Kaler. "Flat-gain L-band Raman-EDFA hybrid optical amplifier for dense wavelength division multiplexed system." IEEE Photonics Technology Letters 25, no. 3, pp: 250252,2013 .

[7] F. J. Duarte,Tunable Laser Optics, $2^{\text {nd }}$ Edition CRC New York,2015. 